Pacific Journal of Mathematics

RELATIVE HERMITIAN MATRICES 


\section{RELATIVE HERMITIAN MATRICES}

\section{Magnus R. Hestenes}

1. Introduction. The purpose of the present paper is to develop a spectral theory for an arbitrary $m \times n$ dimensional matrix $A$, which is analogous to that given in the hermitian case and which reduces to the usual spectral theory when $A$ is hermitian. The theory is centered around the triple product $A B^{*} C$ of matrices of the same dimension. Here $B^{*}$ is the transpose of $B$ in the field of real numbers and the conjugate transpose of $B$ in the field of complex numbers. The matrix $T$ will be said to be elementary in case $T=T T^{*} T$. Elementary matrices play the role of units and in case of vectors are unit vectors. Given an elementary matrix $T$ and a matrix $A$ of the same dimension the matrix $T A^{*} T$ can be considered to be the conjugate transpose of $A$ relative to $T$. If $A=T A^{*} T$, then $A$ is hermitian relative to $T$. The polar decomposition theorem for matrices implies that to each matrix $A$ there is a unique elementary matrix $R$ such that $A$ is hermitian relative to $R, A R^{*}$ is nonnegative hermitian in the usual sense, and $R$ has the same null space as $A$. Every elementary matrix $T$ relative to which $A$ is hermitian is of the form $T=T_{0}+R_{1}-R_{2}$, where $R_{1}+R_{2}=R$ and $T_{0}, R_{1}, R_{2}$ are mutually *-orthogonal. Two matrices $A$ and $B$ are *-orthogonal in case $A B^{*}=0$ and $A^{*} B=0$. A matrix $B$ will be called a section of $A$ if $B$ and $A-B$ are $*$-orthogonal.

If $A$ is hermitian relative to an elementary matrix $T$, it is shown below that $A$ and $T$ can be written as sums of sections

$$
A=A_{1}+\cdots+A_{q}, \quad T=T_{1}+\cdots+T_{q}
$$

such that $A_{i}=\lambda_{i} T_{i}$, where $\lambda_{i}$ is a real number. Moreover these sections can be chosen so that $\lambda_{i} \neq \lambda_{j},(i \neq j)$. If in this event the decomposition is unique. If $A T^{*} \geqq 0$, then $\lambda_{i} \geqq 0$. If in addition $A$ and $T$ have the same null space then $\lambda_{i}>0$. In the event $T$ is the identity, this result gives the usual spectral representation of hermitian matrices.

A matrix $A$ will be said to be normal relative to an elementary matrix $T$ in case $A=A T^{*} T=T T^{*} A, A A^{*} T=T A^{*} A$. In this event the spectral decomposition theorem described above holds, the coefficients $\lambda_{i}$ being complex instead of real.

In the development of the theory the concept of $*$-commutativity of two matrices plays a significant role. The matrices $A$ and $B$ will be said to $*$-commute (see $\S 4$ below) in case $A B^{*}=B A^{*}$ and $A^{*} B=B^{*} A$. If $A$ and $B *$-commute, there is an elementary matrix $T$ relative to

Received April 26, 1960. The preparation of this paper was sponsored by the Office of Naval Research and the Office of Ordnance Research, U.S. Army. Reproduction in whole or in part is permitted for any purpose of the United States Government. 
which they are hermitian. Moreover $A, B T$ can be written as sums of section $A_{i}, B_{i}, T^{i}$ such that $A_{i}=\lambda_{i} T_{i}$ and $B_{i}=\mu_{i} T_{i}$, where $\lambda_{i}$ and $\mu_{i}$ are real. If $\mathfrak{A}$ is a linear class of $m \times n$ dimensional matrices that are hermitian relative to an elementary matrix $T$ in $\mathfrak{A}$ (including $T$ itself) and have the property that the product $A B^{*} C$ is invariant under permutations $A, B$ and $C$, then this class forms an algebra with $A T^{*} B$ as the product of $A$ and $B$. The elements $A$ of $\mathfrak{A}$ are all matrices expressible in the form $A=\lambda_{i} T_{i}+\cdots+\lambda_{q} T_{q}$, where $T_{1}, \cdots, T_{q}$ are suitably chosen sections of $T$. Throughout the paper the reciprocal $A^{*-1}$ of $A^{*}$ plays a role analogous to that of $A$ itself.

The main results obtained in the present paper can be extended to a closed operator $A$ from a Hilbert space $\mathfrak{N}$ to a second Hilbert space $\mathfrak{N}^{\prime}$. Whenever it is convenient to do so, the theorems are stated so as to be valid for operators in Hilbert space. The terminology used has been chosen so as to make this transition as simple as possible. The extension to Hilbert spaces yields a common spectral theory for the gradient of a function and the divergence of a vector field.

2. Terminology and notations. Throughout the following pages matrices will be denoted by capital letters $A, B, C, P, Q, R, \cdots$. The elements can be considered to be real or complex. The conjugate transpose of $A$ will be denoted by $A^{*}$. It will be convenient to consider all matrices to be square, since this can be obtained by the addition of zero-elements. However, this is not essential. The paper is written so as to be valid for rectangular matrices, the equality of the dimensions of two or more matrices being implied by the condition that the operations used should be well defined.

Occasionally we shall use column vectors and row vectors. A column vector will be denoted by $x, y, z, \cdots$. Row vectors are conjugate transposes of column vectors. If $x^{*} x=1$ then $x$ is a unit vector. Given two vectors $x$ and $y$ then $A=y x^{*}$ is a matrix of rank 1 . Every matrix of rank 1 is represented in the form $A=\lambda y x^{*}$, where $\lambda$ is a real number and $x, y$, are unit vectors. In fact $\lambda$ can be taken to be positive. The greek letters, $\alpha, \beta, \gamma, \mu, \cdots$ appearing in the text normally denote real numbers.

A matrix is hermitian if $A^{*}=A$. A hermitian matrix $A$ is nonnegative, written $A \geqq 0$, if $x^{*} A x \geqq 0$ for every column vector $x$. If $A \geqq 0$, there is a unique matrix $B \geqq 0$ such that $B^{2}=A$. The matrix $B$ will be called the square root of $A$. A matrix $E$ will be called a pro-

${ }^{1}$ E. H. Moore, General Analysis, Part I, Mem. Philos. Soc. (1935), p. 197. See also Penrose, “A generalized inverse of matrices", Proc. Cambridge Philos. Soc. 51 (1953), 406413; M. R. Hestenes, "Inversion of matrices by biorthogonalization and related results," $J$. Soc. Ind. Appl. Math. 6 (1958), p. 84; J. von Neumann, On regular rings, Proc. Nat. Acad. Sci. U. S. A. 22 (1936), 707-713. 
jection if $E=E^{*}=E^{2}$. The identity matrix will be denoted by $\mathrm{I}$. "The null space of the matrix $A$ will be denoted by $\mathfrak{N}_{A}$.

To each matrix $A$ there is a unique matrix $B$ such that

$$
A=A A^{*} B=A B^{*} A=B A^{*} A, \quad B=B B^{*} A=B A^{*} B=A B^{*} B .
$$

The matrix $B$ is the reciprocal of $A^{*}$ in the sense of E. H. Moore ${ }^{1}$ and will be called the *-reciprocal of $A$. It is also the conjugate transpose of the reciprocal $A^{-1}$ of $A$. If $A$ is nonsingular, $A^{-1}$ is the inverse of $A$. We shall accordingly use the symbols $A^{*-1}, A^{-1 *}$ for the $*$-reciprocal of $A$. The matrices

$$
E=A^{-1} A=A^{*} A^{*-1}, \quad E^{\prime}=A A^{-1}=A^{*-1} A^{*}
$$

are projections and satisfy the relations

$$
E^{\prime} A=A E=A, \quad E^{\prime} A^{*-1}=A^{*-1} E=A^{*-1} .
$$

They will be called the projections associated with $A$. It should be noted that the reciprocal of $A^{*} A$ is $A^{-1} A^{*-1}$ and that the reciprocal of $A A^{*}$ is $A^{*-1} A^{-1}$. If $A$ is hermitian then $A^{*-1}=A^{-1}$. If $A$ is nonsingular then $A^{-1}$ is the inverse of $A$.

A matrix $R$ will be said to be an elementary matrix in case $R R^{*} R=R$. It is easily seen that $R$ is elementary if and only if $R=R^{*-1}$ or equivalently if and only if $R^{*}=R^{-1}$. If $R$ is elementary so also is $R^{*}$. A projection is an elementary matrix. If $R$ is a hermitian elemetary matrix, then

$$
E_{+}=\frac{1}{2}\left(R^{2}+R\right), \quad E_{-}=\frac{1}{2}\left(R^{2}-R\right)
$$

are projections such that

$$
R=E_{+}-E_{-}, \quad E_{+} E_{-}=E_{-} E_{+}=0, \quad R^{2}=E_{+}+E_{-} .
$$

Conversely, the difference of two projections that are orthogonal is a hermitian elementary matrix.

A matrix $A$ will be said to be hermitan relative to an elementary matrix $R$ if $A=R A^{*} R$. The following result is fundamental.

THEOREM 2.1. Suppose that $A$ is hermitian relative to an elementary matrix $R$. Then $A^{*-1}$ is hermitian relative to $R$ and $A^{*}, A^{-1}$ are hermitian relative to $R^{*}$. Moreover, $\mathfrak{N}_{R} \subset \mathfrak{N}_{A}$ and $\mathfrak{N}_{R^{*}} \subset \mathfrak{R}_{A^{*}}$. The matrices $A$ and $R$ satisfy the further relations

$$
\begin{gathered}
A=R R^{*} A=A R^{*} R=R A^{*} R, \quad R A^{*} A=A A^{*} R=A R^{*} A \\
A^{*} R=R^{*} A, \quad A R^{*}=R A^{*} . \\
\left(A^{*} R\right)^{2}=A^{*} A, \quad\left(A R^{*}\right)^{2}=A A^{*} .
\end{gathered}
$$


(2.3d) $A^{*-1} A^{*} R=R A^{*} A^{*-1}=A R^{*} A^{*-1}=A A^{-1} R=R A^{-1} A=A^{*-1} R^{*} A$.

Since $A=R A^{*} R$ we have $A^{*}=R^{*} A R^{*}$ and $\mathfrak{N}_{R} \subset \mathfrak{N}_{4}, \mathfrak{N}_{R^{*}} \subset \mathfrak{N}_{A^{*} \text {. }}$ Moreover

$$
\begin{gathered}
R R^{*} A=R R^{*} R A^{*} R=R A^{*} R=A=A R^{*} R \\
A R^{*} A=R A^{*} R R^{*} R A^{*} R=R A^{*} R A^{*} R=A A^{*} R=R A^{*} A .
\end{gathered}
$$

It follows that (2.3a) holds. The relations $(2.3 \mathrm{~b})$ and $(2.3 \mathrm{c})$ follow from the computations

$$
\begin{gathered}
A^{*} R=R^{*} A R^{*} R=R^{*} A, \quad R A^{*}=R R^{*} A R^{*}=A R^{*} \\
\left(A^{*} R\right)^{2}=A^{*} R R^{*} A=A^{*} A, \quad\left(A R^{*}\right)^{2}=A R^{*} R A^{*}=A A^{*} .
\end{gathered}
$$

It is easily verified that $R A^{-1} R$ is the reciprocal $R^{*} A R^{*}=A^{*}$. Consequently $A^{*-1}=R A^{-1} R$, that is, $A^{*-1}$ is hermitian relative to $R$. Similarly $A^{-1}$ is hermitian relative to $R^{*}$. The relations (2.3d) follow from (2.3b) and the relations $A A^{-1}=A^{*-1} A^{*}, A^{-1} A=A^{*} A^{*-1}$.

Corollary. Suppose $A=R A^{*} R, R=R R^{*} R$ and set $P=A^{*} R, Q=$ $R A^{*}$. Then

$$
A=R P=Q R, \quad P=R^{*} Q R, \quad Q=R P R^{*} .
$$

The matrix $P$ is nonnegative if and only if $Q$ is nonnegative. Moreover $\mathfrak{N}_{R}=\mathfrak{N}_{A}$ if and only if

$$
R=A^{*-1} R^{*} A
$$

and hence if and only if $\mathfrak{N}_{R^{*}}=\mathfrak{N}_{A^{*}}$.

In view of this result we define a matrix $A$ to be nonnegative hermitian relative to $R$ in case $A=R A^{*} R$ and $A^{*} R \geqq 0$.

Theorem 2.2. Given a matrix $A$ there is a unique elementary matrix $R$ such that $A$ is nonnegatively hermitian relative to $R$ and such that $R=A^{*-1} R^{*} A$. Moreover

$$
R^{*} R=A^{-1} A=A^{*} A^{*-1}, \quad R R^{*}=A A^{-1}=A^{*-1} A^{*} .
$$

Let $P$ be the square root of $A^{*} A$. The matrix $R=A^{*-1} P$ has the properties described in the theorem. Clearly $A^{*} R=A^{*} A^{*-1} P=P \geqq 0$. Moreover

$$
\begin{aligned}
R R^{*} R & =A^{*-1} P^{2} A^{-1} R=A^{*-1}\left(A^{*} A A^{-1} A^{*-1}\right) P=A^{*-1} P=R, \\
R A^{*} R & =R P=A^{*-1} P^{2}=A^{*-1} A^{*} A=A, \\
A^{*-1} R^{*} A & =A^{*-1} P A^{-1} A=A^{*-1} P=R, \\
R^{*} R & =R^{*} A^{*-1} R^{*} A=A^{-1} A=A^{*} A^{*-1}, \\
R R^{*} & =A^{*-1} R^{*} A R^{*}=A^{*-1} A^{*}=A A^{-1} .
\end{aligned}
$$

The uniqueness of $R$ follows from the uniqueness of $P$ as the square: 
root of $A^{*} A$. This proves the theorem.

If $R$ is chosen as described in Theorem 2.2 then the formula (2.4) for $A$ in terms of $P=A^{*} R$ and $R$ is called the polar decomposition ${ }^{2}$ of $A$.

The matrix $R$ described in Theorem 2.2 will be called the elementary matrix associated with $A$.

CoRollary. Let $R$ be the elementary matrix associated with $A$ and let $S$ and $T$ be elementary matrices such that

$$
A=S S^{*} A=A T^{*} T
$$

Then $V=T R^{*} S$ is the elementary operator associated with $B=T A^{*} S$ This follows because

$$
\begin{gathered}
V B^{*} V=T R^{*} S S^{*} A T^{*} T R^{*} S=T R^{*} A R^{*} S=T A^{*} S=B \\
B^{*} V=S^{*} A T^{*} T R^{*} S=S^{*}\left(A R^{*}\right) S \geqq 0 . \\
B^{*-1} V^{*} B=B^{*-1} S^{*} R T^{*} T A^{*} S=B^{*-1} S^{*} R A^{*} S \\
=T A^{-1} S S^{*} A R^{*} S=T A^{-1} A R^{*} S=T R^{*} S=V .
\end{gathered}
$$

As a further result we have

Theorem 2.3. Let $R$ be the elementary matrix associated with $A$. If $A$ is normal so also is $R$. If $A$ is nonnegative hermitian, then $R$ is a projection. If $A$ is hermitian, then $R$ is hermitian and is the difference of two orthogonal projections.

If $A$ is normal, its associated projections $E, E^{\prime}$ coincide. By virtue of (2.6) we have $R R^{*}=R^{*} R$ and $R$ is normal. If $A$ is nonnegative hermitian, then $R=E$. If $A$ is hermitian, let $P$ be the square root of $A^{2}$. Then $A=R P=P R=P R^{*}=R^{*} P$. Consequently $R=R^{*}$, as was to be proved.

The following result is of interest.

TheOREm 2.4. Let $R$ be the elementary matrix associated with $A$. There exists a unique pair of matrices $B, C$ such that

$$
B+C=R, \quad A=B R^{*} C^{*-1}=C^{*-1} R^{*} B
$$

and having $R$ as their associated elementary matrix. The matrices $B$ and $C$ are defined by the formulas

$$
B^{-1}=A^{-1}+R^{*}, \quad C^{-1}=A^{*}+R^{*}
$$

and satisfy the relations

$$
B B^{*} C=B C^{*} B=C B^{*} B, \quad C C^{*} B=C B^{*} C=B C^{*} C
$$

${ }^{2}$ See MacDuffee, C. B., "Theory of Matrices", Ergebnisse der Mathematik und ihrer Grenzgebiete (1933), pp. 77. 


$$
\begin{aligned}
B^{-1} R C^{-1} & =C^{-1} R B^{-1}=B^{-1}+C^{-1} \\
A^{*-1} & =B^{*-1} R^{*} C=C R^{*} B^{*-1}, \quad A^{*}=B^{*} R C^{-1}=C^{-1} R B^{*}, \\
A^{-1} & =B^{-1} R C^{*}=C^{*} R B^{-1} .
\end{aligned}
$$

Since no direct use of this result will be made, its proof will be omitted.

3. *-orthogonality. Two matricies $A$ and $B$ will be said to be: *-orthogonal in case

$$
A^{*} B=B^{*} A=0, \quad A B^{*}=B A^{*}=0 .
$$

Consider now two matrices $A$ and $B$ and let

$$
E=A^{-1} A, \quad E^{\prime}=A A^{-1}, \quad F=B^{-1} B, \quad F^{\prime}=B B^{-1}
$$

be the associated projections. We have the following

Lemma 3.1. Two matrices $A$ and $B$ are *-orthogonal if and only" if

$$
E F=F E=0 \quad E^{\prime} F^{\prime}=F^{\prime} E^{\prime}=0 .
$$

Moreover two matrices $A$ and $B$ are *-orthogonal if and only if their associated elementary matrices $R$ and $S$ are *-orthogonal. Finally $A$ is *-orthogonal to $B$ if and only if $A^{-1 *}$ is *-orthogonal to $B$.

If (3.1) holds, then

$$
\begin{aligned}
E F & =\left(A^{-1} A\right)\left(B^{*} B^{-1 *}\right)=A^{-1}\left(A B^{*}\right) B^{-1 *}=0 \\
E^{\prime} F^{\prime} & =\left(A^{-1 *} A^{*}\right)\left(B B^{-1}\right)=0 .
\end{aligned}
$$

Hence (3.3) holds. The converse follows from the relations $A=A E=E^{\prime} A$, $B=B F=F^{\prime} B$. The last two statements in the lemma follow from the: first.

LemMA 3.2. Let $A$ and $B$ be *-orthogonal matrices and set $C=$ $A+B$. Then

$$
C^{*}=A^{*}+B^{*}, \quad C^{-1}=A^{-1}+B^{-1}, \quad C^{*-1}=A^{*-1}+B^{*-1} .
$$

The rank of $C$ is the sum of the ranks of $A$ and $B$. The elementary. matrix associated with $C$ is the sum $T=R+S$ of the elementary matrices $R, S$ associated with $A, B$, respectively. The matrix $C$ is: elementary if and only if $A$ and $B$ are elementary.

By the use of Lemma 3.1 it is seen that

$$
A^{-1} B=B^{-1} A=0, A B^{-1}=B A^{-1}=0 .
$$

It follows that

$$
\left(A^{-1}+B^{-1}\right) C=A^{-1} A+B^{-1} B=E+F
$$




$$
C\left(A^{-1}+B^{-1}\right)=A A^{-1}+B B^{-1}=E^{\prime}+F^{\prime} .
$$

In view of (3.3) the matrices $G=E+F$ and $G^{\prime}=E^{\prime}+F^{\prime}$ are projections. Moreover, setting $C^{-1}=A^{-1}+B^{-1}$ we have

$$
G^{\prime} C=C G=C, C^{-1} G^{\prime}=G C^{-1}=C^{-1} .
$$

The matrix $C^{-1}$ is therefore reciprocal of $C$ and the relations (3.4) hold. To show that $T=R+S$ is the elementary matrix associated with $C$ observe that $R^{*} B=S^{*} A=0, B R^{*}=A S^{*}=0$, by Theorem 3.1. Hence

$$
\begin{array}{ll}
T^{*} C=R^{*} A+S^{*} B=A^{*} R+B^{*} S=C^{*} T \geqq 0, & C T^{*}=T C^{*} \geqq 0 . \\
T^{*} T=R^{*} R+S^{*} S=E+F=G=C^{-1} C, & T T^{*}=G^{\prime}=C C^{-1},
\end{array}
$$

as was to be proved. The remaining statements in the lemma are easily established.

A matrix $A$ will be said to be a section of a matrix $C$ if there is a matrix $B *$-orthogonal to $A$ such that $C=A+B$. By virtue of the last lemma the elementary matrix $R$ associated with a section $A$ of $C$ is a section of the elementary matrix $T$ belonging to $C$. A section of an elementary matrix is elementary.

LEMMA 3.3. Let $E, E^{\prime}$ be the projections associated with a matrix $A$ and let $F$ and $F^{\prime}$ be projections such that $F^{\prime} A=A F$. Then $E F=F E$, $E^{\prime} F^{\prime}=F^{\prime} E^{\prime}$. Moreover $A_{1}=A F$ is a section of $A$.

Since $A E=A$ it follows that $A F E=F^{\prime} A E=F^{\prime} A=A F$. Consequently

$$
E F E=A^{-1} A F E=A^{-1} A F=E F .
$$

This implies that $E F=F E$. Similarly $E^{\prime} F^{\prime}=F^{\prime} E^{\prime}$. Observe that

$$
\begin{gathered}
F A^{-1}=F E A^{-1}=E F A^{-1}=A^{-1} A F A^{-1}=A^{-1} F^{\prime} A A^{-1}=A^{-1} F^{\prime} E^{\prime}=A^{-1} F^{\prime} \\
F A^{-1} A F=F E F=E F, \quad F^{\prime} A A^{-1} F^{\prime}=F^{\prime} E^{\prime} F^{\prime}=E^{\prime} F^{\prime} .
\end{gathered}
$$

Consequently $A_{1}^{-1}=F A^{-1}=A^{-1} F^{\prime}$ is the reciprocal of $A_{1}=A F=F^{\prime} A$. The projections $E_{1}=E F, E_{0}=E-E_{1}$ are orthogonal as are $E_{1}^{\prime}=E^{\prime} F^{\prime \prime}$, $E_{0}^{\prime}=E^{\prime}-E_{1}^{\prime}$. Consequently $A_{0}=A E_{0}=A-A_{1}=E_{0}^{\prime} A$ is *-orthogonal to $A_{1}$. Since $A=A_{0}+A_{1}$ it follows that $A_{1}$ is a section of $A$, as was to be proved.

Lemma 3.4. $A$ matrix $B$ is a section of $A$ if and only if $A^{*} B=$ $B^{*} B, B A^{*}=B B^{*}$.

Let

$$
F=B^{-1} B, \quad F^{\prime}=B B^{-1} .
$$

If $A^{*} B=B^{*} B, B A^{*}=B B^{*}$

$$
A^{*} F^{\prime}=A^{*} B B^{-1}=B^{*} B B^{-1}=B^{*}
$$




$$
F A^{*}=B^{-1} B A^{*}=B^{-1} B B^{*}=B^{*} \text {. }
$$

Consequently $B=A F=F^{\prime} A$ and $B$ is a section of $A$, by Lemma 3.3. The converse is immediate.

LEMMA 3.5. Let $R$ be an elementary matrix and set $E=R^{*} R$. Let $S=R F$, where $F$ is a projection. Then $S$ is a section of $R$ if and only if $E F=F E$. If $r$ is the rank of $R$ then $R$ is expressible as the sum $R=R_{1}+\cdots+R_{r}$ of $r$ *orthogonal sections of rank 1 .

If $S$ is a section of $R$ then $R^{*} S=S^{*} S=E F=E F E$. Consequently $E F=F E$. Conversely if $E F=F E$ then

$$
\begin{aligned}
& S^{*} S=F R^{*} R F=F E F=E F=R^{*} R F=R^{*} S \\
& S S^{*}=R F^{2} R^{*}=R F R^{*}=S R^{*} .
\end{aligned}
$$

It follows from Lemma 3.4 that $S$ is a section of $R$.

In order to prove the last statement in the theorem suppose that $R \neq 0$ and choose a unit vector $x$ such that $E x=x$. Then $E_{1}=x x^{*}$ is a projection that commutes with $E$. Hence $R_{1}=R E_{1}$ is a section of $R$ of rank 1. Moreover $R-R_{1}$ is a section of $R$ of rank $r-1$ and is *-orthogonal to $R_{1}$. If $R-R_{1} \neq 0$ it has a section $R_{2}$ of rank 1 . Clearly $R_{2}$ is *-orthogonal to $R_{1}$ and $R-R_{1}-R_{2}$ is a section of $R$ of rank $r-2$. By a repetition of this argument it is seen that $R$ is expressible as the sum of $r *$-orthogonal sections, as was to be proved.

4. *-commutativity. Given two matrices $A$ and $B$ the products $A^{*} B$ and $A B^{*}$ can be considered to be two types of *-products of $A$ and $B$. If these $*$-products are unaltered upon interchanging $A$ and $B$, that is, if

$$
A^{*} B=B^{*} A, \quad A B^{*}=B A^{*},
$$

then $A$ and $B$ will be said to $*$-commute. It should be noted that $A$ and $B *$-commute if and only if $A^{*} B$ and $A B^{*}$ are hermitian in the usual sense. As a first result we have

LEMMA 4.1. If $A$ and $B$ *-commute and

$$
E=A^{-1} A, \quad E^{\prime}=A A^{-1}, \quad F=B^{-1} B, \quad F^{\prime}=B B^{-1},
$$

then $F^{\prime} A=A F, E^{\prime} B=B E$ and $E F=F E, E^{\prime} F^{\prime}=F^{\prime} E^{\prime}$.

For if (4.1) holds then, since $F^{\prime} B=B F=B$, we have

$$
\begin{gathered}
F^{\prime} A F=B^{*-1} B^{*} A F=B^{*-1} A^{*} B F=B^{*-1} A^{*} B=B^{*-1} B^{*} A=F^{\prime} A \\
F^{\prime} A F=F^{\prime} A B^{*} B^{*-1}=F^{\prime} B^{*} A B^{*-1}=B^{*} A B^{*-1}=A B^{*} B^{*-1}=A F .
\end{gathered}
$$

Consequently $A F=F^{\prime} A$. Similarly $E^{\prime} B=B E$. In view of Lemma 3.3 the relations $E F=F E, E^{\prime} F^{\prime}=F^{\prime} E^{\prime}$ hold. 
THeOREM 4.1. Two matrices $A$ and $B *$-commute if and only if they are expressible in the form $A=A_{0}+A_{1}, B=B_{0}+B_{1}$, where $A_{0}$ is *-orthogonal to $A_{1}$ and $B, B_{0}$ is *-orthogonal to $B_{1}$ and $A, A_{1}$ and $B_{1}$ *-commute and have the same associated projections

$$
E_{1}=A_{1}^{-1} A_{1}=B_{1}^{-1} B_{1}, \quad E_{1}^{\prime}=A_{1} A_{1}^{-1}=B_{1} B_{1}^{-1} .
$$

If $A$ and $B *$-commute, then, by Lemmas 4.1 and 3.3, the matrices $A_{1}=A F, B_{1}=B E$ are sections of $A$ and $B$ respectively and have $E_{1}=$ $E F, E_{1}^{\prime}=E^{\prime} F^{\prime}$ as their associated projections. Moreover $A_{0}=A-A_{1}$ has $E-E F, E^{\prime}-E^{\prime} F^{\prime}$ as its projections and hence is $*$-orthogonal to $B$ and $A_{1}$ and hence also to $B_{1}$ and $B_{0}=B-B_{1}$. Similarly $B_{0}$ is *orthogonal to $B_{1}, A, A_{0}$ and $A_{1}$. The converse is immediate and the lemma is proved.

Corollary. Suppose that $A$ and $B$ *-commute. Then $A$ and $A^{*-1}$ *-commute with $B$ and $B^{*-1}$. Moreover $A^{*}, A^{-1} *$-commute with $B^{*}$ and $B^{-1}$.

We shall see later that their associated matrices $R, S *$-commute with $A, B, R$, and $S$.

THEOREM 4.2. Let $R$ be the elementary matrix associated with a matrix $A$ and let $S$ be an elementary matrix that *-commutes with $A$. Then $S$ *-commutes with $R$. Moreover $A, R, S$ are expressible uniquely as sums and differences

$$
A=A_{0}+A_{+}+A_{-}, \quad R=R_{0}+R_{+}+R_{-}, \quad S=S_{0}+R_{+}-R_{-}
$$

of *-orthogonal matrices such that the matrices $R_{0}, R_{+}, R_{-}$are the elementary matrices associated with $A_{0}, A_{+}, A_{-}$respectively and such that $S_{0}$ is *-orthogonal to $A_{0}$ and $R_{0}$. Conversely if $A, R, S$ can be decomposed in this manner the $A$ and $R$ *-commute with $S$.

By virtue of the last theorem $A$ and $S$ can be expressible uniquely as the sum of *-orthogonal sections $A=A_{0}+A_{1}, S=S_{0}+S_{1}$ such that $A_{1}$ and $S_{1}$ have the same associated projections, $S_{0}$ being *-orthogonal to $A$ and $A_{0}$ being *-orthogonal to $S$. The elementary matrix $R$ associated with $A$ is expressible in the form $R=R_{0}+R_{1}$, where $R_{0}$ and $R_{1}$ are the elementary matrices associated with $A_{0}$ and $A_{1}$ respectively. In view of these remarks we can restrict ourselves to the case in which $A_{0}=0, S_{0}=0$ and $R_{0}=0$. Then

$$
E=A^{-1} A=R^{*} R=S^{*} S, \quad E^{\prime}=A A^{-1}=R R^{*}=S S^{*} .
$$

Since $A^{*} S$ is self-adjoint, its associated elementary matrix $T$ is the difference $T=E_{+}-E_{-}$of two orthogonal projections $E_{+}$and $E_{-}$whose sum is $E$. The matrix $A^{*} S T$ is nonnegative and self-adjoint. It follows from Theorem 2.1 that $R=S T$. The matrices $R_{+}=R E_{+}, R_{-}=R E_{-}$ are $*$-orthogonal elementary matrices such that 


$$
R=R E=R_{+}+R_{-}, \quad S=R T=R_{+}-R_{-} .
$$

Since $A R^{*}$ and $A S^{*}$ are hermitian it follows that the matrices

$$
A R_{+}^{*}=\frac{1}{2} A\left(R^{*}+S^{*}\right), \quad A R_{-}^{*}=\frac{1}{2} A\left(R^{*}-S^{*}\right)
$$

are hermitian and orthogonal. Moreover they are nonnegative because of the relations

$$
0 \leqq A R^{*}=A R_{+}^{*}+A R_{-}^{*} .
$$

The elementary matrices $R_{+}$and $R_{-}$are accordingly the elementary matrices associated with $A_{+}=A E_{+}$and $A_{-}=A E_{-}$respectively. It is clear that $A_{+}$and $A_{-}$are *-orthogonal and that $A=A_{+}+A_{-}$. The matrices $A, R, S$ are therefore expressible in the form (4.4). The converse is immediate and the theorem is established.

Corollary 1. Two elementary matrices $R$ and $S$ *-commute if and only if there exist mutually matrices $R_{0}, R_{+}, R_{-}, S_{0}$ such that $R=R_{0}+R_{+}+R_{-}, S=S_{0}+R_{+}-R_{-}$. Moreover this decomposition is unique.

COROLlary 2. If the matrix $S$ appearing in Theorem 4.2 is of rank 1 then the decomposition (4.4) of A takes the simpler form

$$
A=\mu S+A_{0}
$$

where $\mu$ is a real number and $A_{0}$ is *-orthogonal to $S$.

For in this case two of the matrices $S_{0}, R_{+}, R_{-}$are zero since $S$ has rank 1. If $S=S_{0}$, then (4.5) holds with $\mu=0$. If $S=R_{+}$, then $R_{-}=A_{-}=0$ and $A_{+}$is of rank 1 . Since $S^{*} A_{+}$is a nonegative hermitian matrix of rank 1 it follows that $A_{+}$is of the form $A_{+}=\mu S$, where $\mu>0$. If $S=-R_{-}$, then $A_{-}$is of the form $A_{-}=\mu S$ with $\mu<0$.

COROLlaRY 3. If $S_{1}, \cdots, S_{r}$ are r mutually *-orthogonal elementary matrices of rank 1 that *-commute with $A$, then $A$ is expressible in the form

$$
A=\mu_{1} S_{1}+\cdots+\mu_{r} S_{r}+A_{0}
$$

where $\mu_{1}, \cdots, \mu_{r}$ are real numbers and $A_{0}$ is *-orthogonal to each $S_{i}(i=1, \cdots, r)$ and hence to $S_{1}+\cdots+S_{r}$.

This result follows from Corollary 2 by induction. At the $k$ th step one applies Corollary 2 with $A$ replaced by $A-\mu_{1} S_{1}-\cdots-\mu_{k-1} S_{k-1}$ and with $S=S_{k}$.

THEOREM 4.3. If a matrix $A$ *-commutes with every section of an elementary matrix $S$ than $A$ is expressible in the form

$$
A=\mu S+A_{0}
$$


where $\mu$ is a real number and $A_{0}$ is *-orthogonal to $S$.

If $S$ has rank 1, the theorem holds by virtue of Corollary 2 to Theorem 4.2. If $S$ is of rank $r>1$, then, by Lemma 3.5, $S$ is expressible in the form $S=S_{1}+\cdots+S_{r}$ where $S_{1}, \cdots, S_{r}$ are mutually *-orthogonal elementary matrices. Consequently $A$ is expressible in the form (4.6). It remains to show that $\mu_{1}=\mu_{2}=\cdots=\mu_{r}$. To this end choose unit vectors $x_{i}$ and $y_{i}$ such that $S_{i}=x_{i} y_{i}^{*}$. Then for $i \neq j$ the vector $x_{i}$ is orthogonal to $x_{j}$ and $y_{i}$ is orthogonal to $y_{j}$. Let $\alpha$ and $\beta$ be two nonnull real numbers such that $\alpha^{2}+\beta^{2}=1$ and set

$$
x=\alpha x_{i}+\beta x_{j}, \quad y=\alpha y_{i}+\beta y_{j} .
$$

Then $T=x y^{*}$ is a section of $S$ and is $*$-orthogonal to $S_{k}$ if $k \neq i, k \neq j$. The matrix

$$
A^{*} T=\left(\mu_{i} S_{i}^{*}+\mu_{j} S_{j}^{*}\right) T=\left(\mu_{i} \alpha y_{i}+\mu_{j} \beta y_{j}\right) y^{*}
$$

is hermitian if and only if $\mu_{i}=\mu_{j}$, that is $T *$-commutes with $A$ if and only if $\mu_{i}=\mu_{j}$. This completes the proof of the theorem.

THEOREM 4.4. A matrix $A$ *-commutes with an elementary matrix $S$ and has no nonnull section *-orthogonal to $S$ if and only if $A=S A^{*} S$.

This result is easily established. The condition that $A=S A^{*} S$, when $S=I$ is the condition that $A$ be hermitian. Accordingly one can consider the condition $A=S A^{*} S$ to be an extension of the concept of a matrix being hermitian.

In the complex domain we have the following:

Corollary. If $A$ is a matrix and $S$ is an elementary matrix such that $S S^{*} A=A S^{*} S=A$, then $B=(1 / 2)\left(A+S A^{*} S\right)$ and $C=(1 / 2 i)\left(A-S A^{*} S\right)$ *-commute with $S$. Moreover, $A=B+i C$.

5. Principal values and principal sections of matrices. Here and elsewhere the symbol $\|y\|$ denotes the length or norm of the vector $y$. By the norm $\|A\|$ of a matrix $A$ will be meant the least upper bound of the quantity $\|A x\|$ for all unit vectors $x$. If $R$ is the elementary matrix associated with $A$, then $\|A\|$ is equal to the least upper bound of $\|A x\|$ subject to the condition $\|R x\|=1$. As is well known there is a unit vector $x$ such that $\|A x\|=\|A\|$. For such a vector $x$ we have $\|R x\|=1$ also. It is well known that $\|A\|=\left\|A^{*}\right\|$. If $A \neq 0$ then $\left\|A^{-1}\right\|=\left\|A^{*-1}\right\|$ is equal to the least number $m$ such that $\|A x\| \geqq(1 / m)\|R x\|$.

Theorem 5.1. Let $R$ be the elementary matrix associated with $A$. Given a positive number $\lambda$ there exists a unique decomposition

$$
A=A_{+}+A_{0}+A_{-}, \quad R=R_{+}+R_{0}+R_{-}
$$


on $A$ and $R$ into mutually *-orthogonal sections such that $R_{+}, R_{0}, R_{-}$, $R_{+}-R_{-}$are the elementary matrices associated with $A_{+}, A_{0}, A_{-}, A-\lambda R$ respectively. Moreover $A_{0}=\lambda R_{0}$ and

$$
\begin{aligned}
& \left\|A_{+} x\right\|>\lambda\left\|R_{+} x\right\| \quad \text { whenever } R_{+} x \neq 0 \\
& \left\|A_{-} x\right\|<\lambda\left\|R_{-} x\right\| \quad \text { whenever } R_{-} x \neq 0 .
\end{aligned}
$$

If $\lambda \geqq\|A\|$, then $A_{+}=R_{+}=0$. If $1 / \lambda \leqq\left\|A^{*-1}\right\|$ then $A_{-}=R_{-}=0$.

In order to prove this result let $B=A-\lambda R$ and let $S$ be the associated elementary matrix. Since $R *$-commutes with $A$ and $R$, it *-commutes with $B$ and hence also with $S$. Since $S$ *-commutes with $B$ and $R$ it follows that $S *$-commutes with $A$. Applying Theorem 4.2 to $A, R, S$ and to $B, R, S$ it is seen that they are expressible as sums

$$
\begin{aligned}
A & =A_{+}+A_{0}+A_{-}, & R & =R_{+}+R_{0}+R_{-} \\
B & =B_{+}+B_{0}-B_{-}, & S & =R_{+}+S_{0}-R_{-} .
\end{aligned}
$$

of $*$-orthogonal matrices such that $R_{+}$is the elementary matrix associated with $A_{+}$and $B_{+}, R_{-}$is the elementary matrix associated with $A_{-}$and $B_{-}, R_{0}$ is the elementary matrix associated with $A_{0}$. It is clear that $B_{0}=S_{0}=0$ since every matrix that is $*$-orthogonal to $A$ and $R$ is also *-orthogonal to $B$ and $S$. From the relation $A=B+\lambda R$ it follows that

$$
A_{0}=\lambda R_{0}, \quad A_{+}=B_{+}+\lambda R_{+}, \quad A_{-}=B_{-}+\lambda R_{-} .
$$

Consequently

$$
R_{+}^{*} A_{+}=R_{+}^{*} B_{+}+\lambda E_{+}, \quad R_{-}^{*} A_{-}+R_{-}^{*} B_{-}=\lambda E_{-} .
$$

Since these matrices are nonnegative and hermitian, it is seen that (5.2) holds. The last statement in the theorem follows from the relations (5.2).

THEOREM 5.2. A nonull matrix $A$ and its associated elementary matrix $R$ have unique decompositions of the form

$$
A=\lambda_{1} R_{1}+\cdots+\lambda_{k} R_{k}, \quad R=R_{1}+\cdots+R_{k}
$$

into $*$-orthogonal sections, where $\lambda_{1}, \cdots, \lambda_{k}$ are distinct positive numbers.

In order to prove this result let $\lambda_{1}=\|A\|$. By virtue of the last theorem the matrices $A$ and $R$ are expressible as sums

$$
A=\lambda_{1} R_{1}+B, \quad R=R_{1}+S
$$

of *-orthogonal sections with $\|B\|<\lambda_{1}$. If $B \neq 0$, choose $\lambda_{2}=\|B\|$ and, by Theorem 5.1, again, $B$ and $S$ are sums

$$
B=\lambda_{2} R_{2}+C, \quad S=R_{2}+T
$$

of sections. Proceeding in this manner one obtains the representation 
described in the theorem.

The numbers $\lambda_{1}, \cdots, \lambda_{k}$ appearing in the last theorem will be called the principal values of $A$ and the matrices $A_{1}=\lambda_{1} R_{1}, \cdots, A_{k}=\lambda_{k} R_{k}$ will be called the principal sections of $A$. The rank of $A_{i}$ will be called the multiplicity of $\lambda_{i}$ as a principal value of $A$. It is easily seen that a number $\lambda>0$ is a principal value of $A$ of multiplicity $m$ if and only if it is an eigenvalue of $R^{*} A$ (or $A R^{*}$ ) of multiplicity $m$. Similarly a number $\lambda>0$ is a principal value of $A$ if and only if $\lambda^{2}$ is an eigenvalue of $A^{*} A$ (or of $A A^{*}$ ) and the multiplicities are the same. It is easily seen that a number $\lambda$ is a principal value if and only if the equation $A x=\lambda R x$ has a solution $x$ with $R x \neq 0$.

It follows from the last theorem that the principal values of $A$ are the norms of the nonnull sections of $A$ and in particular the norms of the principal sections of $A$. A section $B$ of rank 1 of the principal section $A_{i}=\lambda_{i} R_{i}$ of $A$ is expressible in the form $B=\lambda_{i} y x^{*}$, where $x$ and $y$ are unit vectors. A vector of the form $\alpha x(\alpha \neq 0)$ will be called a principal vector of $A$ and a vector of the form $\beta y(\beta \neq 0)$ will be called a reciprocal principal vector of $A$ corresponding to the principal value $\lambda_{i}$. It is easily seen that $x$ is an eigenvector of $A^{*} A$ and that $y$ is an eigenvector of $A A^{*}$.

Theorem 5.3. A matrix $A$ is normal if and only if its principal sections are normal. A matrix $A$ is hermitian if and only if its principal sections are hermitian. $A$ matrix $A$ is hermitian and nonnegative if and only if its principal sections are hermitian and nonnegative.

In order to prove this result let $A$ and $R$ be represented in the form (5.3) with $\lambda_{1}>\lambda_{2}>\cdots>\lambda_{k}$. Let

$$
B=A-\lambda_{k} R=\left(\lambda_{1}-\lambda_{k}\right) R_{1}+\cdots+\left(\lambda_{k-1}-\lambda_{k}\right) R_{k-1} .
$$

If $A$ is normal so also are $R$ and $B$. It follows that $S=R_{1}+R_{2}+\cdots+R_{k-1}$, the elementary matrix belonging to $B$, is also normal. This implies that $R_{k}=R-S$ and $A_{k}=\lambda_{k} R_{k}$ are normal. The same argument applied to $B$ shows that $R_{k-1}$ and $A_{k-1}=\lambda_{k-1} R_{k-1}$ are normal. It follows that each princiqal section $A_{i}=\lambda_{i} R_{i}(1 \leqq i \leqq k)$ of $A$ is normal whenever $A$ is normal. Conversely if $A_{1}, \cdots, A_{k}$ are normal so also is $A$. This proves the first statement in the theorem. The second statement can be proved similarly. The third statement is an easy consequence of the second and the concept of nonnegativeness.

THEOREM 5.4. Let $X$ and $Y$ be elementary matrices such that the relation

$$
Y Y^{*} A=A X X^{*}=A
$$

holds for a given matrix $A$. Then $B=Y^{*} A X$ has the same principal 
values as those of $A$ and have the same multiplicities. If $R$ is the associated elementary matrix for $A$, then $S=Y^{*} R X$ is the associated elementary matrix for $B$. There exist elementary matrices $X$ and $Y$ such that $B$ is a nonnegative diagonal matrix and $R=Y X^{*}$.

Setting $S_{i}=Y^{*} R_{i} X, S=S_{1}+\cdots+S_{k}$ it is found that $B=$ $\lambda_{1} S_{1}+\cdots+\lambda_{k} S_{k}$. The ranks of $S_{i}$ and $R_{i}$ coincide and $S_{i} *$-commutes with $S_{j}(i \neq j)$. The last statement can be obtained by selecting a maximal set of mutually orthogonal principal vectors $x_{1}, \cdots, x_{r}$ of $A$ of unit length and setting $y_{h}=R x_{h}(h=1, \cdots, r)$. Let $X$ be the matrix whose first $r$ column vectors are $x_{1}, \cdots, x_{r}$ and the remaining vectors are null vectors. The matrix $Y=R X$ has $y_{1}, \cdots, y_{r}$ as its first $r$ column vectors. It is easily seen that $X$ and $Y$ are elementary matrices of rank $r$ having the properties described in the theorem. In fact the nonzero elements of $B=Y^{*} A X$ are the principal values of $A$. One could restrict $X$ to have only $r$ columns if one so desires. One could modify $X$ and $Y$ so as to be nonsingular. In this event we would have $R=Y E X^{*}$, where $E=R^{*} R$. In either event the column vectors of $C=A X$ are mutually orthogonal and the lengths of the nonnull column vectors of $C$ are the principal values of $A$. This fact can be used to devise a modified Jacobi method for finding the principal values of $A$. A discussion of a method of this type will be given by the author in a forthcoming paper.

6. Further properties of $*$-commutativity. Throughout the present section let $A$ denote a given matrix and let $R$ be its associated elementary matrix. Let

$$
A=\lambda_{1} R_{1}+\cdots+\lambda_{k} R_{k}, \quad R=R_{1}+\cdots+R_{k}
$$

be its decomposition into principal sections, given in Theorem 5.2. As before we set

(6.2) $A_{i}=\lambda_{i} R_{i}, \quad E_{i}=R_{i}^{*} R_{i}, \quad E_{i}^{\prime}=R_{i} R_{i}^{*}, \quad E=R^{*} R, \quad E^{\prime}=R R^{*}$.

The first result to be established is given in the following.

THEOREM 6.1. If a matrix $B$ *-commutes with $A$, then it $*$-commutes with every matrix of the form

$$
\mathrm{C}=\nu_{1} R_{1}+\cdots+\nu_{k} R_{k}
$$

where $\nu_{1}, \cdots, \nu_{k}$ are real numbers. In particular $B *$-commutes with $R$ and with each principal section $A_{j}(j=1, \cdots, k)$. The matrix $B$ is expressible uniquely as the sum

$$
B=B_{0}+B_{1}+\cdots+B_{k}
$$

of *-orthogonal sections such that $B_{i}$ is *-orthogonal to $A_{j}(j \neq i)$ and 
$B_{i}(i>0) *$-commutes with $A_{i}$.

In order to prove this result we may suppose that the principal sections of $A$ have been ordered so that $\lambda_{1}<\lambda_{2}<\cdots<\lambda_{k}$. Recall that, by virture of the corollary to Theorem 4.1, the matrix $B$ not only *-commutes with $A$ but also with

$$
A^{*-1}=\left(1 / \lambda_{1}\right) R_{1}+\cdots+\left(1 / \lambda_{k}\right) R_{k} .
$$

It follows that $B *$-commutes with

$$
C_{2}=A-\lambda_{1}^{2} A^{*-1}=\lambda_{22} R_{2}+\cdots+\lambda_{k 2} R_{k}
$$

where $\lambda_{i 2}=\lambda_{i}-\lambda_{1}\left(\lambda_{1} / \lambda_{i}\right)>0(i=2, \cdots, k)$. Moreover $\lambda_{i 2}<\lambda_{j 2}(i<j)$, as one readily verifies. Using the recursion formula

$$
C_{j+1}=C_{j}-\lambda_{j j}^{2} C_{j}^{*-1}, \quad \lambda_{j+1, i}=\lambda_{j i}-\frac{\lambda_{j j}^{2}}{\lambda_{j i}} \quad(i=j+1, \cdots, k)
$$

one obtains matrices of the form

$$
C_{j}=\lambda_{j j} R_{j}+\cdots+\lambda_{k j} R_{k} \quad(j=2, \cdots, k)
$$

that *-commute with $B$. Moreover each $R_{i}$ is a linear combination of $C_{1}=A, C_{2}, \cdots, C_{k}$. It follows that $B *$-commutes with each of the sections $R_{1}, \cdots, R_{k}$ of $R$. Consequently $B *$-commutes with any matrix $C$ of the form (6.3).

In order to prove that $B$ is of the form (6.4) it follows from Theorem 4.1 with $A$ replaced $A_{i}$ that $B$ is expressible in the form $B=C_{i}+B_{i}$ where $C_{i}$ is *-orthogonal to $A_{i}$ and $B_{0}=B-\left(B_{1}+\cdots+B_{k}\right)$ is *-orthogonal to each $B_{j}$ and $A_{j}(j=1, \cdots, k)$ and hence also to $A$. This completes the proof of the theorem.

THEOREM 6.2. If a matrix $B$ *-commutes with every section of $A$, then $B$ is expressible in the form

$$
B=B_{0}+\mu_{1} R_{1}+\cdots+\mu_{k} R_{k}
$$

where $\mu_{1}, \cdots, \mu_{k}$ are real numbers and $B_{0}$ is *-orthogonal to $A$.

Let $B_{0}, B_{1}, \cdots, B_{k}$ be the sections of $B$ given in (6.4). Since every section of $A_{i}$ and hence every section of $R_{i} *$-commutes with $B$ and hence with $B_{i}$ it follows from Theorem 4.3 that $B_{i}$ is of the form $B_{i}=\mu_{i} R_{i}+B_{i 0}$, where $\mu_{i}$ is a real number and $B_{i 0}$ is $*$-orthogonal to $R_{i}$. It is clear from the definition of $B_{i}$ that $B_{i 0}$ must be zero. This proves the theorem.

Theorem 6.3. A matrix $B$ *-commutes with $A$ if and only if it *-commutes with $R$ and $A R^{*} B=B R^{*} A$.

If $B *$-commutes with $A$, then $B *$-commutes with $R$ and

$$
A R^{*} B=A B^{*} R=B A^{*} R=B R^{*} A .
$$


Conversely, suppose that $B *$-commutes with $R$ and that $A R^{*} B=B R^{*} A$. Then $A^{*} R B^{*}=B^{*} R A^{*}$ and

$$
\begin{aligned}
& A^{*} B=R^{*} R A^{*} B=R^{*} A R^{*} B=R^{*} B R^{*} A=B^{*} R R^{*} A=B^{*} A \\
& A B^{*}=R R^{*} A B^{*}=R A^{*} R B^{*}=R B^{*} R A^{*}=B R^{*} R A^{*}=B A^{*},
\end{aligned}
$$

as was to be proved.

Corollary. If $A$ is a positive definite hermitian matrix, then $B$ *-commutes with $A$ if and only if $B$ is hermitian and $A B=B A$.

This result is immediate since $R=I$ for a positive definite matrix.

It should be observed that if $A$ is hermitian but not definite, then there are nonhermitian matrices that $*$-commute with $A$. For example the matrices

$$
A=\left(\begin{array}{rr}
1 & 0 \\
0 & -1
\end{array}\right), \quad B=\left(\begin{array}{rr}
1 & -1 \\
1 & 1
\end{array}\right)
$$

*-commute even though $B$ is not hermitian. However $A$ is elementary and $B=A B^{*} A$, that is, $B$ is hermitian relative to $A$.

THEOREM 6.4. Let $A$ be a matrix and let $T$ be an elementary matrix such that $T A^{*} T=A$. Let $B$ be a matrix that $*$-commutes with $T$. Then $B$ *-commutes with $A$ if and only if $A T^{*} B=B T^{*} A$.

The proof is similar to that of the last theorem and will be omitted.

TheOREM 6.5. Given a matrix $B$ that *-commutes with $A$ there exists a set of mutually *-orthogonal elementary matrices $T_{1}, \cdots, T_{q}$ with the property that $A$ and $B$ are expressible in the form

$$
A=\alpha_{1} T_{1}+\cdots+\alpha_{q} T_{q}, \quad B=\beta_{1} T_{1}+\cdots+\beta_{q} T_{q}
$$

where $\alpha_{1}, \cdots, \alpha_{q}$ are equal numbers, $\beta_{1}, \cdots, \beta_{q}$ are equal numbers and $\alpha_{i}=\alpha_{j}, \beta_{i}=\beta_{j}$ holds only in case $i=j$. If $\alpha_{i} \neq 0$, then $\left|\alpha_{i}\right|$ is a principal value of $A$. Similarly if $\beta_{i} \neq 0$ then $\left|\beta_{i}\right|$ is a principal value.

It is clear that $T_{i}$ may be replaced by $-T_{i}$ in the theorem. The matrices $T_{1}, \cdots, T_{q}$ will be uniquely determined if, for example, one requires that $\beta_{i} \geqq 0$ and that $\alpha_{i}>0$ if $\beta_{i}=0$.

Let

$$
A=\lambda_{1} R_{1}+\cdots+\lambda_{k} R_{k}, \quad B=\mu_{1} S_{1}+\cdots+\mu_{m} S_{m}
$$

be the decompositions of $A$ and $B$ respectively into principal sections. Recall that by virtue of Corollary 1 to Theorem 4.2 the matrices $R_{i}$ and $S_{j}$ are expressible as sums

$$
R_{i}=R_{i j 0}+R_{i j+}+R_{i j_{-}}, \quad S_{j}=S_{i j 0}+R_{i j+}-R_{i j_{-}} .
$$

Let $T_{1}, \cdots, T_{p}$ be all non-null elementary matrices $R_{i j+}$ and $R_{i j-}$ obtained. 
in this manner. Adjoin to these the maximal nonnull section of each $R_{i}$ that is *-orthogonal to $S$ and the maximal nonnull section of each $S_{j}$ that is *-orthogonal to $R$. The elementary matrices $T_{1}, \cdots, T_{q}$ obtained in this manner are $*$-orthogonal and have the property that each $R_{i}$ and $S_{f}$ is expressible uniquely in the form

$$
R_{i}=\rho_{i 1} T_{1}+\cdots+\rho_{i q} T_{q}, \quad S_{j}=\sigma_{j 1} T_{1}+\cdots+\sigma_{j q} T_{q}
$$

where $\rho_{i k}=0$ if $T_{k}$ is *-orthogonal to $R_{i}, \rho_{i h}=1$ if $T_{h}$ is a section of $R_{i}, \rho_{i h}=-1$ if $-T_{h}$ is a section of $R_{i}, \sigma_{j h}=0$ if $T_{h}$ is *-orthogonal to $S_{j}, \sigma_{j h}=1$ if $T_{h}$ is a section of $S_{j}$ and $\sigma_{j h}=-1$ if $-T_{h}$ if a section of $S_{j}$. Combining this result with (6.7) one obtains (6.6). The last statement of the theorem follows from the construction just made.

As a consequence of this result we have

THEOREM 6.6. Let $T$ be an elementary matrix and let $A$ be a matrix satisfying the condition $A=T A^{*} T$. Then $A$ and $T$ can be represented uniquely as the sum

$$
A=\alpha_{1} T_{1}+\cdots+\alpha_{q} T_{q}, \quad T=T_{1}+\cdots+T_{q}
$$

of mutually $*$-orthogonal matrices such that $\alpha_{i} \neq \alpha_{j}(i \neq j)$.

This result follows from the last theorem with $B=T$ and the condition that $\beta_{i} \geqq 0$. Since no nonnull section of $A$ is $*$-orthogonal to $T$ we have $\beta_{i}=1$, and the theorem follows.

If $T$ is the identity then $A$ is hermitian and $\alpha_{1}, \cdots, \alpha_{q}$ are the eigenvalues of $A$. The rank of $T_{i}$ is the multiplicity of $\alpha_{i}$ as an eigenvalue of $A$. This result suggests that we call $\alpha_{1}, \cdots, \alpha_{q}$ the principal values or eigenvalues of $A$ relative to $T$, the rank of $T_{i}$ being the multiplicity of $\alpha_{i}$.

As an extension of the last theorem we have

THEOREM 6.7. Let $T$ be an elementary matrix and let $A$ and $B$ be *-commutative matrices such that $A=T A^{*} T$ and $B=T B^{*} T$. There is a unique decomposition

$$
T=T_{1}+\cdots+T_{q}
$$

into sections such that $A$ and $B$ are representable in the form

$$
A=\alpha_{1} T_{1}+\cdots+\alpha_{q} T_{q}, \quad B=\beta_{1} T_{1}+\cdots+\beta_{q} T_{q}
$$

where $\alpha_{i}=\alpha_{j}, \beta_{i}=\beta_{j}$ holds only in case $i=j$.

The proof of this result can be made by a simple modification of the proof of the last two theorems and will be omitted.

In the complex domain we have the following

Corollary 1. Let $T$ be an elementary matrix and let $C$ be a 
matrix such that $T T^{*} C=C T^{*} T=C$ and $T C^{*} C=C C^{*} T$. Then $C$ and $T$ have unique decompositions

$$
C=\gamma_{1} T_{1}+\cdots+\gamma_{q} T_{q}, \quad T=T_{1}+\cdots+T_{q}
$$

in sections, where $\gamma_{1}, \cdots, \gamma_{q}$ are distinct complex numbers.

For, by the corollary to Theorem 4.4, the matrix $C$ is expressible in the form $C=A+i B$, where $A$ and $B *$-commute with $T$. From the relation $T C^{*} C=C C^{*} T$ it is found that $A T^{*} B=B T^{*} A$ and hence that $A$ and $B *$-commute. The corollary follows from the last theorem with $\gamma_{\jmath}=\alpha_{j}+i \beta_{j}(j=1, \cdots, q)$.

If $T=I$, the result described in the corollary yields the spectral decomposition for normal matrices.

By the use of an argument like that given in the proof of Theorem 5.4 one obtains the further result described in the following

CoRollary 2. Let $A$ and $B$ be *-commutative matrices and let $T$ be an elementary matrix such that $A=T A^{*} T$ and $B=T B^{*} T$. There exist elementary matrices $X$ and $Y$ such that

$$
Y Y^{*} T=T X X^{*}=T
$$

and such that $Y^{*} A X, Y^{*} B X$ are diagonal matrices. If $C=A+i B$, then $Y^{*} C X$ is also a diagonal matrix.

7. Certain classes of matrices. Let $\mathscr{S}(A)$ be the class of matrices $B$ that $*$-commute with $A$ and have no non-null section that is $*$-orthogonal to $A$. Let $\mathscr{C}(A)$ be all matrices $B$ such that $\mathscr{S}(A)$ is a subclass of $\mathscr{S}(B)$. It is clear that $A$ is in $\mathscr{C}(A)$. We have the following

THEOREM 7.1. Let $R$ be the elementary matrix associated with $A$ and let

$$
A=\lambda_{1} R_{1}+\cdots+\lambda_{k} R_{k}, \quad R=R_{1}+\cdots+R_{k}
$$

be the decomposition of $A$ into principal sections. The class $\mathscr{C}(A)$ consists of all matrices $B$ that are expressible in the form

$$
B=\mu_{1} R_{1}+\cdots+\mu_{k} R_{k}
$$

where $\mu_{1}, \cdots, \mu_{k}$ are real numbers. If $B$ is in $\mathscr{C}(A)$ so also is $B^{*-1}$ and its associated elementary matrix $S$.

This result follows from Theorems 6.1 and 6.2.

Corollary. If $B$ is in $\mathscr{C}(A)$ then $\mathscr{C}(B) \subset \mathscr{C}(A)$. Moreover $\mathscr{C}(B)=\mathscr{C}(A)$ if and only if $B$ has the same number of distinct principal values as $A$.

As a further result we have

TheOREM 7.2. If $B, C, D$ are matrices in $\mathscr{C}(A)$ so also is $M=B C^{*} D$. 
In fact

$$
B C^{*} D=B D^{*} C=C B^{*} D=C D^{*} B=D B^{*} C=D C^{*} B .
$$

The relations (6.2) follow from the fact that $B, C, D *$-commute with each other. Observe that $M^{*}=B^{*} C D^{*}$. If $N$ is a matrix in $\mathscr{C}(A)$ then

$$
N^{*} M=N^{*} B C^{*} D=B^{*} N C{ }^{*} D=B^{*} C N^{*} D=B^{*} C D^{*} N=M^{*} N .
$$

Similarly $N M^{*}=M N^{*}$. This proves the theorem.

In view of the formula (7.1) one obtains the following

THEOREM 7.3. Let

$$
B=\mu_{1} R_{1}+\cdots+\mu_{k} R_{k}, \quad C=\nu_{1} R_{1}+\cdots+\nu_{k} R_{k}
$$

be two matrices in $\mathscr{C}(A)$. Then

$$
\alpha B+\beta C=\left(\alpha \mu_{1}+\beta \nu_{1}\right) R_{1}+\cdots+\left(\alpha \mu_{k}+\beta \nu_{k}\right) R_{k}
$$

is in $\mathscr{C}(A)$ for every pair of real numbers $\alpha$ and $\beta$. If we define the product of $B$ and $C$ by the formula $B \cdot C=B R^{*} C$, then

$$
B \cdot C=\mu_{1} \nu_{1} R_{1}+\cdots+\mu_{k} \nu_{k} R_{k}
$$

is in $\mathscr{C}(A)$ and the usual laws of algebra hold. In particular $B \cdot R=$ $R \cdot B=B$. Given a polynomial

$$
p_{m}(\lambda)=\alpha_{0}+\alpha_{1} \lambda+\cdots+\alpha_{m} \lambda^{m}
$$

with real coefficients set

$$
p_{m}(A, R)=\alpha_{0} A^{(0)}+\alpha_{1} A^{(1)}+\cdots+\alpha_{\hat{n}} A^{(n)},
$$

where $A^{(0)}=R, A^{(1)}=A, A^{(h)}=A \cdot A^{(h-1)}$. Then the polynomial

$$
p_{m}(A, R)=p_{m}\left(\lambda_{1}\right) R_{1}+\cdots+p_{m}\left(\lambda_{k}\right) R_{k}
$$

in $A$ relative to $R$ is in $\mathscr{C}(A)$. Conversely every matrix $B$ in $\mathscr{C}(C)$ is expressible as a real polynominal in $A$ relative to $R$ of degree $\leqq k-1$. There is a unique polynomial $p_{k}(\lambda)$ with leading coefficient $\alpha_{k}=1$ such that $p_{k}(A, R)=0$.

The first three statements in the lemma are immediate. The matrix $B$ is given by the relative polynomial $p_{k-1}(A, R)$ whose coefficients $\alpha_{0}, \alpha_{1}, \cdots, \alpha_{k-1}$ are given by the solutions of the equations

$$
\alpha_{0} \lambda_{1}^{h}+\alpha_{1} \lambda_{2}^{h}+\cdots+\alpha_{k-1} \lambda_{k}^{h}=\mu_{h} \quad(h=0,1, \cdots, k-1) .
$$

Finally the polynomial $p_{k}(\lambda)$ described in the last statement in the theorem is the polynomial of degree $k$ whose roots are $\lambda_{1}, \lambda_{2}, \cdots, \lambda_{k}$.

Corollary. On the class $\mathscr{C}(A)$ the norm $\|B\|$ satisfies the rela- 
tion $\|B \cdot C\| \leqq\|B\|\|C\|$.

As a final property of the classes $\mathscr{C}(A)$ we have

Theorem 7.4. If $B$ is in $\mathscr{S}(A)$ there is a matrix $C$ such that $A$ and $B$ are in $\mathscr{C}(C)$.

This result follows from Theorem 6.5 with

$$
C=T_{1}+2 T_{2}+3 T_{3}+\cdots q T_{q} .
$$

Let $T$ be a given elementary matrix and let $A$ be a matrix in $\mathscr{S}(T)$. Let $\mathscr{S}(A, T)$ be all matrices in $\mathscr{S}(T)$ that *-commute with $A$. Let $\mathscr{C}(A, T)$ be all matrices $B$ in $\mathscr{S}(A, T)$ such that $\mathscr{S}(A, T) \subset \mathscr{S}(B, T)$. If $T$ is the elementary matrix $R$ associated with $A$ then $\mathscr{C}(A, T)=\mathscr{C}(A)$. Let

$$
A=\alpha_{1} T_{1}+\cdots+\alpha_{q} T_{q}, \quad T=T_{1}+\cdots T_{q}
$$

be the decomposition of $A$ and $T$ given in Theorem 6.6. Then, as is easily seen, the class $\mathscr{C}(A, T)$ consists of all matrices of the form

$$
B=\beta_{1} T_{1}+\cdots+\beta_{q} T_{q}
$$

where $\beta_{1}, \cdots, \beta_{q}$ are real numbers. If we set

$$
C=T_{1}+2 T_{2}+\cdots+q T_{q}
$$

then the class $\mathscr{C}(A, T)$ coincides with the class $\mathscr{C}(C)$. Consequently the results stated above are applicable to the class $\mathscr{C}(A)$. If $T$ is the identity, then $A$ is hermitian and $\mathscr{C}(A, T)$ consists of all hermitian matrices that commute with every hermitian matrix that commutes with $A$.

Consider now an elementary matrix $T$ and let $\mathfrak{R}(T)$ be the class of all matrices $A$ such that $T T^{*} A=A T^{*} T=A$ and $A A^{*} T=T A^{*} A$. Given a matrix $A$ in $\mathfrak{R}(T)$ let $\mathfrak{M}(A, T)$ be the class of all matrices $B$ in $\mathfrak{R}(T)$ such that $A T^{*} B=B T^{*} A$ and $A B^{*} T=T B^{*} A$. If $B$ is in $\mathfrak{M}(A, T)$ then $B A^{*} T=T A^{*} B$ also. Moreover, $T B^{*} T$ is in $\mathfrak{M}(A, T)$. Let $\mathscr{B}(A, T)$ be the class of all matrices $B$ such that $\mathfrak{M}(B, T) \supset \mathfrak{M}(A, T)$. In view of Corollary 1 to Theorem 6.7 the matrices $A$ and $T$ are expressible uniquely in the form

$$
A=\alpha_{1} T_{1}+\cdots+\alpha_{q} T_{q}, \quad T=T+\cdots+T_{q},
$$

where $\alpha_{1}, \alpha_{2}, \cdots, \alpha_{q}$ are distinct complex numbers. It is not difficult to show a matrix $B$ is in $\mathscr{B}(A, T)$ if and only if it is expressible in the form

$$
B=\beta_{1} T_{1}+\cdots+\beta_{q} T_{q},
$$

where $\beta_{1}, \cdots, \beta_{q}$ are complex numbers. If $B$ and $C$ are in $\mathscr{B}(\dot{A}, T)$ so 
also are $\alpha B+\beta C$, where $\alpha$ and $\beta$ are complex numbers. Moreover, the product $B \cdot C=B T^{*} C$ is in $\mathscr{B}(A, T)$. If polynomials of $A$ relative to $T$ are defined as before, but with complex coefficients, it is seen that the class $\mathscr{B}(A, T)$ is made up of all polynomials of $A$ relative to $T$ of degree $\leqq q-1$. Again we have the relation $\|B \cdot C\| \leqq\|B\|\|C\|$. These results generalize the corresponding theory for normal matrices.

University of California, Los ANGELES 



\section{PACIFIC JOURNAL OF MATHEMATICS}

\section{EDITORS}

Ralph S. Phillips

Stanford University

Stanford, California

F. H. BrownelL

University of Washington

Seattle 5 , Washington
A. L. Whiteman

University of Southern California Los Angeles 7. California

L. J. PAIGE

University of California

Los Angeles 24, California

ASSOCIATE EDITORS
E. F. BECKENBACH
D. DERRY
H. L. ROYDEN
E. G. STRAUS
T. M. CHERRY
M. OHTSUKA
E. SPANIER
F. WOLF

\section{SUPPORTING INSTITUTIONS}

UNIVERSITY OF BRITISH COLUMBIA

CALIFORNIA INSTITUTE OF TECHNOLOGY

UNIVERSITY OF CALIFORNIA

MONTANA STATE UNIVERSITY

UNIVERSITY OF NEVADA

NEW MEXICO STATE UNIVERSITY

OREGON STATE COLLEGE

UNIVERSITY OF OREGON

OSAKA UNIVERSITY

UNIVERSITY OF SOUTHERN CALIFORNIA
STANFORD UNIVERSITY

UNIVERSITY OF TOKYO

UNIVERSITY OF UTAH

WASHINGTON STATE COLLEGE,

UNIVERSITY OF WASHINGTON

AMERICAN MATHEMATICAL SOCIETY

CALIFORNIA RESEARCH CORPORATION

HUGHES AIRCRAFT COMPANY

SPACE TECHNOLOGY LABORATORIES

NAVAL ORDNANCE TEST STATION

Printed in Japan by International Academic Printing Co., Ltd., Tokyo, Japan

Reprinted 1966 in the United States of America 


\section{Pacific Journal of Mathematics}

\section{Vol. 11, No. $1 \quad$ November, 1961}

A. A. Albert, Generalized twisted fields ............................ 1

Richard Arens, Operational calculus of linear relations ................... 9

John Herbert Barrett, Disconjugacy of a self-adjoint differential equation of the fourth order ....................................... 25

Paul Richard Beesack, Hardy's inequality and its extensions ............... 39

Julius Rubin Blum and David Lee Hanson, On invariant probability measures.

II .............................................

Robert Allen Bonic, Symmetry in group algebras of discrete groups.......... 73

R. Creighton Buck, Multiplication operators ...................... 95

Jack Gary Ceder, Some generalizations of metric spaces ................. 105

Meyer Dwass, Random crossings of cumulative distribution functions ......... 127

Albert Edrei, Wolfgang H. J. Fuchs and Simon Hellerstein, Radial distribution and

deficiencies of the values of a meromorphic function ............... 135

William Cassidy Fox, Harmonic functions with arbitrary local singularities ..... 153

Theodore Thomas Frankel, Manifolds with positive curvature ............... 165

Avner Friedman, A strong maximum principle for weakly subparabolic

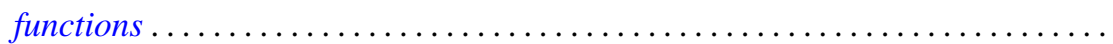

Watson Bryan Fulks and J. O. Sather, Asymptotics. II. Laplace's method for

multiple integrals ......................................

Adriano Mario Garsia and Eugene Richard Rodemich, An embedding of Riemann

surfaces of genus one ..................................... 193

Irving Leonard Glicksberg, Weak compactness and separate continuity......... 205

Branko Grünbaum, On a conjecture of H. Hadwiger .................. 215

Frank J. Hahn, On the action of a locally compact group on $E_{n} \ldots \ldots \ldots \ldots \ldots . . \ldots 221$

Magnus R. Hestenes, Relative hermitian matrices ..................... 225

G. K. Kalisch, On similarity invariants of certain operators in $L_{p} \ldots \ldots \ldots \ldots .247$

Yitzhak Katznelson and Walter Rudin, The Stone-Weierstrass property in Banach

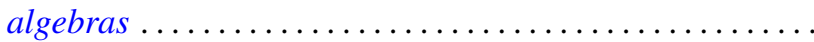

Samir A. Khabbaz, The subgroups of a divisible group $G$ which can be represented as intersections of divisible subgroups of $G \ldots \ldots \ldots \ldots \ldots \ldots \ldots \ldots \ldots . \ldots \ldots 7$

Marvin Isadore Knopp, Construction of a class of modular functions and forms .......................................... 275

Charles Alan McCarthy, Commuting Boolean algebras of projections .......... 295

T. M. MacRobert, Transformations of series of E-functions ................ 309

Heinz Renggli, An inequality for logarithmic capacities ................. 313

M. S. Robertson, Applications of the subordination principle to univalent functions .......................................... 315

David Sachs, Partition and modulated lattices ..................... 325

Frank S. Scalora, Abstract martingale convergence theorems ............... 347

Elbert A. Walker, Torsion endomorphic images of mixed Abelian groups ........ 375

Morgan Ward, The prime divisors of Fibonacci numbers................. 379

Charles R. B. Wright, On the nilpotency class of a group of exponent four....... 387 